\title{
Genetic Predisposition Factors in Renal Disorders
}

\section{Rad IA*}

Department of Medical Genetics, Motahari Teaching Hospital, Urmia University of Medical Sciences, Urmia, West Azerbaijan, Iran

*Corresponding author: Isa Abdi Rad, MD PhD, Department of Medical Genetics, Motahari Teaching Hospital, Urmia University of Medical Sciences, Kashani Street, Urmia, West Azerbaijan, Iran, Tel: +98-44-32240166/+914 440 5829; E-mail: isaabdirad@yahoo.com

Received date: July 11, 2017; Accepted date: July 12, 2017; Published date: July 14, 2017

Copyright: @ 2017 Rad IA, et al. This is an open-access article distributed under the terms of the Creative Commons Attribution License, which permits unrestricted use, distribution, and reproduction in any medium, provided the original author and source are credited.

Citation: Rad IA (2017) Genetic Predisposition Factors in Renal Disorders. J Kidney 3: e109. doi:10.4172/2472-1220.1000e109

\section{Editorial}

Genetic susceptibility plays an important role in the development of many renal disorders. Revealing the etiologic basis and predisposing factors of renal disorders have impacts on the prevention of these prevalent disorders which have a global health burden with a high economic cost. Prevention of renal diseases also has impact on the prevention of other disorders since renal diseases can cause other health problems such as heart disease. The fundamental basis of prevention is determination of predisposing factors especially genetic factors, because by recognition of genetic basis of the disease, prevention can be applied in the prenatal period.

Some renal disorders are single gene disorders with obvious Mendelian inheritance such as polycystic kidney diseases. Autosomaldominant polycystic kidney disease (ADPKD) is the fourth most common cause for renal transplantation and is responsible for up to $10 \%$ of end-stage renal disease. [1] Mutations in the PKD1 and PKD2 genes result in ADPKD. Autosomal-recessive polycystic kidney disease (ARPKD) is childhood-onset polycystic kidney disease with the incidence of 1 in 20,000 live births and is an important cause of chronic kidney disease in children and is caused by mutations in PKHD1 gene. [2]

In some renal disorders many genes can be involved in the pathogenesis of a single pathologic entity. Focal segmental glomerulosclerosis (FSGS) can be caused by mutations in more than 20 podocyte-specific genes such as NPHS1, NPHS2, WT-1, LAMB2, CD2AP, TRPC6, ACTN4 and INF2 [3]. In these conditions clarification of genotype-phenotype relationships would be important in targeted therapy and prognostication.

Renal disorders sometimes influenced by genetic factors, however, not with Mendelian pattern of inheritance. In these conditions, a renal disorder can be a multi-factorial disorder or shows susceptibility due to specific genetic polymorphisms. Nephropathy resulted from diabetes mellitus or hypertension is a multi-factorial disorder, that is, caused by interaction of environmental factors with multiple genes or genetic susceptibility that determine the time of onset and the rate of progression of the renal disease. For example, a polymorphism in the CNDP1 gene, which encodes the enzyme carnosinase-1, determines susceptibility to develop diabetic nephropathy [4].

Renal disorders sometimes are part of genetic syndromes or disorders with or without dysmorphic features. For example in tyrosinemia type I which is associated with liver dysfunction and renal tubular dysfunction, due to mutations in $F A H$ gene [5], there is no dysmorphism. On the other hand, there is constellation of dysmorphic features associated with renal disorder in many genetic syndromes such as branchio-oto-renal (BOR) syndrome. Dysmorphic features of BOR syndrome include prominent ears, low-set ears, auricular pits and tags associated with middle, and inner ear anomalies, branchial fistulae and cysts. Renal malformations which may occur in BOR syndrome include renal agenesis, multiple renal cysts, renal dysplasia, ectopic kidneys, hydronephrosis, small kidneys, double ureters, urinary reflux and ureteral atresia. Mutations in three genes EYA1, SIX1, and SIX5 can result in BOR syndrome. [6]

In brief, the genetic predisposition factors have a crucial impact on the renal diseases. These factors may be a monogenic condition leading to a known renal disease, or a multi-factorial condition and polymorphisms that determine time of onset and progression rate of a renal disease. And sometimes a renal disorder is a component of a genetic syndrome. Current advances in whole genome analysis can reveal the role of genetic variants and polymorphisms in the development of different kidney diseases.

\section{References}

1. Spithoven E, Kramer A, Meijer E, Orskov B, Wanner C, et al. (2014) Renal replacement therapy for autosomal dominant polycystic kidney disease (ADPKD) in Europe: Prevalence and survival-an analysis of data from the ERA-EDTA Registry. Nephrol Dial Transplant 29: 15-25.

2. Adeva M, El-Youssef M, Rossetti S, Kamath PS, Kubly V, et al. (2006) Clinical and molecular characterization defines a broadened spectrum of autosomal recessive polycystic kidney disease (ARPKD). Medicine (Baltimore) 85: 1-21.

3. Chen YM, Liapis H (2015) Focal segmental glomerulosclerosis: Molecular genetics and targeted therapies. BMC Nephrol 16: 101.

4. Janssen B, Hohenadel D, Brinkkoetter P, Peters V, Rind N, et al. (2005) Carnosine as a protective factor in diabetic nephropathy-association with a leucine repeat of the carnosinase gene CNDP1. Diabetes 54: 2320-2327.

5. St-Louis M, Tanguay RM (1997) Mutations in the fumarylacetoacetate hydrolase gene causing hereditary tyrosinemia type I: Overview. Hum Mutat 9: 291-299.

6. Krug P, Morinière V, Marlin S, Koubi V, Gabriel HD, et al. (2011) Mutation screening of the EYA1, SIX1 and SIX5 genes in a large cohort of patients harboring branchio-oto-renal syndrome calls into question the pathogenic role of SIX5 mutations. Hum Mutat 32: 183-190. 